Open Access

\title{
Comparison of an automatic analysis and a manual analysis of conjunctival microcirculation in a sheep model of haemorrhagic shock
}

Philip-Helge Arnemann ${ }^{1 * \dagger} \mathbb{D}$, Michael Hessler ${ }^{1 \dagger}$, Tim Kampmeier $^{1}$, Andrea Morelli ${ }^{2}$, Hugo Karel Van Aken ${ }^{1}$, Martin Westphal', Sebastian Rehberg ${ }^{3}$ and Christian Ertmer $^{1}$

\section{* Correspondence:}

arnemann@anit.uni-muenster.de ${ }^{\dagger}$ Equal contributors

'Department of Anaesthesiology, Intensive Care and Pain Therapy, Muenster University Hospital, Albert-Schweitzer-Campus 1, Building A1, 49149 Muenster, Germany

Full list of author information is available at the end of the article

\begin{abstract}
Background: Life-threatening diseases of critically ill patients are known to derange microcirculation. Automatic analysis of microcirculation would provide a bedside diagnostic tool for microcirculatory disorders and allow immediate therapeutic decisions based upon microcirculation analysis.

Methods: After induction of general anaesthesia and instrumentation for haemodynamic monitoring, haemorrhagic shock was induced in ten female sheep by stepwise blood withdrawal of $3 \times 10 \mathrm{~mL}$ per kilogram body weight. Before and after the induction of haemorrhagic shock, haemodynamic variables, samples for blood gas analysis, and videos of conjunctival microcirculation were obtained by incident dark field illumination microscopy. Microcirculatory videos were analysed (1) manually with AVA software version 3.2 by an experienced user and (2) automatically by AVA software version 4.2 for total vessel density (TVD), perfused vessel density (PVD) and proportion of perfused vessels (PPV). Correlation between the two analysis methods was examined by intraclass correlation coefficient and Bland-Altman analysis.

Results: The induction of haemorrhagic shock decreased the mean arterial pressure (from $87 \pm 11$ to $40 \pm 7 \mathrm{mmHg} ; p<0.001$ ); stroke volume index (from $38 \pm 14$ to $20 \pm$ $5 \mathrm{ml} \cdot \mathrm{m}^{-2} ; p=0.001$ ) and cardiac index (from $2.9 \pm 0.9$ to $1.8 \pm 0.5 \mathrm{~L} \cdot \mathrm{min}^{-1} \cdot \mathrm{m}^{-2} ; p<0$. 001 ) and increased the heart rate (from $72 \pm 9$ to $87 \pm 11 \mathrm{bpm} ; p<0.001$ ) and lactate concentration (from $0.9 \pm 0.3$ to $2.0 \pm 0.6 \mathrm{mmol} \cdot \mathrm{L}^{-1} ; p=0.001$ ). Manual analysis showed no change in TVD $\left(17.8 \pm 4.2\right.$ to $\left.17.8 \pm 3.8 \mathrm{~mm}^{*} \mathrm{~mm}^{-2} ; p=0.993\right)$, whereas PVD (from $15.6 \pm 4.6$ to $11.5 \pm 6.5 \mathrm{~mm}^{*} \mathrm{~mm}^{-2} ; p=0.041$ ) and PPV (from $85.9 \pm 11.8$ to $62.7 \pm 29.6 \% ; p=0.017)$ decreased significantly. Automatic analysis was not able to identify these changes. Correlation analysis showed a poor correlation between the analysis methods and a wide spread of values in Bland-Altman analysis.

(Continued on next page)
\end{abstract}


(Continued from previous page)

Conclusions: As characteristic changes in microcirculation during ovine haemorrhagic shock were not detected by automatic analysis and correlation between automatic and manual analyses (current gold standard) was poor, the use of the investigated software for automatic analysis of microcirculation cannot be recommended in its current version at least in the investigated model. Further improvements in automatic vessel detection are needed before its routine use.

Keywords: Microcirculation, Analysis, Automatic, Manual, Haemorrhagic shock

\section{Background}

Life-threatening diseases of critically ill patients are often accompanied by changes in microvascular perfusion [1-5], and the persistence of microcirculatory abnormalities is associated with poor outcome [3, 4].

In recent years, methods such as sidestream dark field imaging (SDF) [6] or incident dark field illumination imaging (IDF) [7] were developed to allow a direct observation of the microcirculation at the bedside. Directly monitoring the microcirculation is a powerful diagnostic tool and may help to better understand the individual problems of patients and the effects of haemodynamic therapy in the critical care setting [8]. Based on the availability of microcirculatory monitoring at the bedside and accumulating evidence, treatment decisions may be made on the basis of microvascular parameters in the near future $[9,10]$.

A major shortcoming constraining the widespread evaluation of the microcirculation at the bedside is the prolonged time necessary for the quantitative analysis. The current gold standard and method of choice is a time-consuming manual analysis that includes plotting of each vessel and quantification of flow in every individual vessel in a video performed by an experienced user [11]. A software conducting this analysis automatically could help to establish the microcirculation as a tool for "point-of-care" diagnosis and decision-making.

The aim of this study was to compare a software for an automatic analysis of key microcirculatory variables, such as total vessel density (TVD), perfused vessel density (PVD) and proportion of perfused vessels (PPV), with a manual analysis, which is the current gold standard [11]. An additional intention was to correlate the results of these two methods.

\section{Methods}

\section{Study design}

Ten sheep were anaesthetized and instrumented as described below. After reaching shock time point and the respective measurements, data acquisition for the present study ends, and the sheep were randomised for an interventional study of haemorrhagic shock, whose data is not part of the present analysis. At the end of that study, the sheep were killed with an intravenous injection of $4 \mathrm{mg} / \mathrm{kg}$ propofol and of $200 \mathrm{~mL}$ potassium chloride solution (7.45\%).

\section{Anaesthesia and instrumentation}

General anaesthesia was induced in ten female sheep (species Ovis orientalis aries) by intramuscular bolus injection of $0.3 \mathrm{mg} \cdot \mathrm{kg}^{-1}$ midazolame and $10 \mathrm{mg} \cdot \mathrm{kg}^{-1} \mathrm{~S}$-ketamine. 
Following endotracheal intubation, balanced anaesthesia was maintained by inhalational isoflurane with an expiratory fraction of $1.2 \mathrm{vol} \%$ as well as a continuous infusion of $0.3 \mathrm{mg} \cdot \mathrm{kg}^{-1} \cdot \mathrm{h}^{-1}$ midazolame and $1 \mathrm{mg} \cdot \mathrm{kg}^{-1} \cdot \mathrm{h}^{-1} \mathrm{~S}$-ketamine. The sheep were ventilated targeting an end-tidal carbon dioxide partial pressure of $35 \pm 5 \mathrm{mmHg}$.

A pulse contour cardiac output catheter $\left(5-\mathrm{Fr} \mathrm{PiCCO}^{\mathrm{mw}}\right.$ catheter, Pulsion Medical Systems, Munich, Germany) was placed in the left femoral artery to obtain haemodynamic variables. A central venous line was inserted into the right jugular vein to administer drugs and to conduct thermodilution as well as a 7.5-Fr catheter in the left jugular vein for blood withdrawal. A Foley catheter was placed in the urinary bladder.

Afterwards, the sheep were turned into prone position and were allowed to recover for a period of $30 \mathrm{~min}$ before the experimental protocol was started.

\section{Experimental protocol}

Following baseline measurements ("baseline" time point), $3 \times 10 \mathrm{~mL}$ blood per kilogram body weight was withdrawn stepwise over a period of 5 min each. After each of the three blood withdrawals, there was a recovery period of $30 \mathrm{~min}$. The resulting $30 \mathrm{~mL}$ per kg body weight of withdrawn blood equals approximately $50-60 \%$ of the total blood volume in the sheep [12]. If mean arterial pressure (MAP) decreased below $30 \mathrm{mmHg}$ during blood withdrawal, the current step of withdrawal was stopped immediately resulting in less than $10 \mathrm{~mL}$ per $\mathrm{kg}$ body weight for the individual withdrawal.

After 3 cycles of blood withdrawal, each followed by 30 min of recovery time, shock time was defined after the last recovery time. At shock time point, haemodynamic and microcirculatory measurements were performed.

\section{Measurements}

At both time points (baseline and shock), systemic haemodynamic variables and conjunctival microcirculation were measured and arterial blood samples were obtained for the blood gas analysis.

Haemodynamic measurements comprised MAP, stroke volume index (SVI) and cardiac index (CI). SVI and CI were obtained by threefold bolus thermodilution using the $\mathrm{PiCCO}^{\text {ix }}$ system. The mean of the three measurements was documented. Also, the heart rate was read from the haemodynamic monitoring tool.

Conjunctival microcirculation was measured in five different conjunctival positions at both measurement time points. Measurements were conducted using an IDF camera $\left(\right.$ CytoCam $^{\mathrm{Tu}}$, Braedius Medical BV, Huizen, The Netherlands). The obtained videos were reviewed for quality according to recommendations by Massey et al. [13] and discarded if necessary.

The remaining high-quality videos were analysed (1) by an experienced user blinded for the study protocol using a manual analysis software (AVA software version 3.2, Microvision Medical, Amsterdam, The Netherlands) and (2) a software including an automatic video analysis of microcirculatory videos (AVA software version 4.2, Microvision Medical, Amsterdam, The Netherlands).

With both programs, the TVD, PVD and the PPV were determined in each video according to an independent expert consensus conference to characterise microcirculatory perfusion [11]. 
Manual analysis with AVA software version 3.2 comprised the manual drawing of each vessel and assigning a diameter and flow score to each vessel and has been described thoroughly before [14]. After the manual processing, the AVA 3.2 software calculated the above-mentioned parameters. In the AVA software version 4.2, the software conducts the process of detecting the vessels and flow automatically. As the consensus conference recommended including only vessels with a diameter of less than $20 \mu \mathrm{m}$ in the microcirculatory analysis, this threshold was used in both analysis methods.

\section{Statistical analysis}

Statistical analysis was performed with IBM SPSS statistics software version 22 (IBM, Armonk, New York, USA). All data are presented as mean with standard deviation, unless otherwise stated.

Variables were tested to confirm the equality of variances by Levene's test, and Kolmogorov-Smirnov test was used to confirm normal distribution. For comparisons between time points, paired sample $t$ test was used. Asymptotic two-sided $p$ values smaller than 0.05 were assumed as statistically significant.

For the comparison of manual and automatic analyses of sheep, conjunctival microcirculation intraclass correlation coefficient (ICC) was determined [15]. The ICC for normally distributed, continuous values is presented with $95 \%$ confidence intervals as a measure of dispersion. Values below 0.40 are considered as "poor" agreement, between 0.40 and 0.59 as "fair" agreement, between 0.60 and 0.74 as "good" agreement and for greater than 0.74, the level of agreement is "excellent" as suggested by Cicchetti [16].

In addition, the method suggested by Bland and Altman [17] was used to assess the agreement between the two analysis methods. According to Bland and Altman, the mean difference of the two values (manual and automatic) for each video was plotted against the average of those two values. The mean bias (95\% confidence interval) was calculated as well as the limits of agreement (LOA) as 1.96-fold of the standard deviation of the mean bias.

\section{Results}

Haemodynamic variables and arterial blood gas analysis

$24.3 \pm 4.5 \mathrm{~mL} / \mathrm{kg}$ of blood was withdrawn. In seven sheep, blood withdrawal was stopped due to predefined safety measures. Withdrawal of blood resulted in a decrease in MAP, CI and SVI and an increase in HR from baseline to shock time point. In addition, an increase in arterial lactate concentration as well as a reduction of arterial haemoglobin concentration was found (Table 1).

\section{Microcirculatory variables at baseline and in haemorrhagic shock}

Of the 100 videos taken of sheep conjunctival microcirculation, 85 met the predefined quality criteria [13]. Microcirculatory variables were examined regarding differences between baseline and shock for each of the two analysis methods (Table 2). Manual analysis showed a significant decrease in PVD and a significant reduction of PPV in shock compared to baseline, while TVD remained constant. Contrarily, no statistically significant differences could be demonstrated between baseline and shock for any of the variables with the automatic analysis (Additional files 1 and 2). 
Table 1 Haemodynamic variables and arterial blood gas analysis

\begin{tabular}{|c|c|c|c|}
\hline Variable & Baseline $(n=10)$ & Shock $(n=10)$ & $p$ value \\
\hline MAP [mmHg] & $87 \pm 11$ & $40 \pm 7$ & $<0.001^{\S}$ \\
\hline $\mathrm{HR}$ [bpm] & $72 \pm 9$ & $87 \pm 11$ & $0.003^{\S}$ \\
\hline $\mathrm{Cl}\left[\mathrm{L} \mathrm{min}^{-1} \cdot \mathrm{m}^{-2}\right]$ & $2.9 \pm 0.9$ & $1.8 \pm 0.5$ & $<0.001^{\S}$ \\
\hline SVI $\left[\mathrm{mL} \cdot \mathrm{m}^{-2}\right]$ & $38 \pm 14$ & $20 \pm 5$ & $0.001^{\S}$ \\
\hline $\mathrm{Hb}\left[\mathrm{g} \cdot \mathrm{dL}^{-1}\right]$ & $8.6 \pm 0.7$ & $8.0 \pm 0.5$ & $0.003^{\S}$ \\
\hline Lactate $\left[\mathrm{mmo} \cdot \mathrm{L}^{-1}\right]$ & $0.9 \pm 0.3$ & $2.0 \pm 0.6$ & $0.001^{\S}$ \\
\hline
\end{tabular}

All values are presented as mean \pm standard deviation. Lactate $=$ arterial lactate concentration MAP mean arterial pressure, $H R$ heart rate, $\mathrm{Cl}$ cardiac index, SVI stroke volume index, $\mathrm{Hb}$ arterial haemoglobin concentration

${ }^{\S}$ Significant difference between baseline and shock

\section{Results of intraclass correlation coefficient and Bland-Altman analysis}

The ICC, calculated for the complete data set $(n=85)$ and for the subgroups baseline $(n=41)$ and shock $(n=44)$, showed a poor agreement [16] for all examined variables (Table 3).

In addition, the Bland-Altman analysis revealed a bias between manual and automatic analyses for all tested variables with wide LOA (Table 4). Figure 1 demonstrates the respective Bland-Altman plots for TVD and PVD $(n=85)$.

\section{Discussion}

The main results of the present study are that ovine haemorrhagic shock induced by blood withdrawal caused a reduction in PVD and PPV if analysis of microcirculation was conducted manually (with AVA software version 3.2). Automatic analysis (by AVA software version 4.2) was not able to reproduce the findings of the gold standard method. Accordingly, there was a wide variation of values, and Bland-Altman analysis as well as ICC revealed a poor correlation between manual and automatic analyses.

A reliable automatic analysis of microcirculation would be associated with multiple advantages. First, this method would be markedly faster than manual analysis thus allowing bedside application. Based upon our own experiences, automatic analysis by AVA software version 4.2 takes approximately 3 to 4 min while manual analysis by an experienced user takes about 20 min per video. Second, based on the faster analysis at the bedside, monitoring of the microcirculation would become applicable for immediate therapeutic decisions. Third, the analysis would be independent of the user's

Table 2 Microcirculatory variables

\begin{tabular}{lllll}
\hline Analysis method & Variable & Baseline $(n=10)$ & Shock $(n=10)$ & $p$ value \\
\hline Manual analysis & TVD $\left[\mathrm{mm}^{*} \mathrm{~mm}^{-2}\right]$ & $17.8 \pm 4.2$ & $17.8 \pm 3.8$ & 0.993 \\
& PVD $\left[\mathrm{mm}^{*} \mathrm{~mm}^{-2}\right]$ & $15.6 \pm 4.6$ & $11.5 \pm 6.5$ & $0.041^{\S}$ \\
& PPV [\%] & $85.9 \pm 11.8$ & $62.7 \pm 29.6$ & $0.017^{\S}$ \\
Automatic analysis & TVD $\left[\mathrm{mm}^{*} \mathrm{~mm}^{-2}\right]$ & $10.6 \pm 1.4$ & $11.0 \pm 1.9$ & 0.460 \\
& PVD $\left[\mathrm{mm}^{*} \mathrm{~mm}^{-2}\right]$ & $10.2 \pm 1.4$ & $10.8 \pm 1.9$ & 0.330 \\
& PPV [\%] & $96.1 \pm 6$. & $97.9 \pm 3.7$ & 0.372 \\
\hline
\end{tabular}

All values are presented as mean \pm standard deviation

$T V D$ total vessel density, PVD perfused vessel density, PPV proportion of perfused vessels

${ }^{\S}$ Significant difference between baseline and shock 
Table 3 Intraclass correlation coefficient between manual and automatic analyses

\begin{tabular}{lllll}
\hline Variable & Data set & Number & ICC [95\% Cl] & Agreement \\
\hline TVD $\left[\mathrm{mm}^{*} \mathrm{~mm}^{-2}\right]$ & All videos & 85 & $-0.267[-0.949-0.176]$ & Poor \\
& Baseline & 41 & $-0.069[-1.01-0.430]$ & Poor \\
& Shock & 44 & $-0.568[-1.874-0.144]$ & Poor \\
PVD $\left[\mathrm{mm}^{*} \mathrm{~mm}^{-2}\right]$ & All videos & 85 & $-0.219[-0.875-0.208]$ & Poor \\
& Baseline & 41 & $-0.013[-0.899-0.460]$ & Poor \\
& Shock & 44 & $-0.379[-1.538-0.247]$ & Poor \\
PPV [\%] & All videos & 85 & $-0.074[-0.651-0.302]$ & Poor \\
& Baseline & 41 & $0.274[-0.361-0.613]$ & Poor \\
& Shock & 44 & $-0.193[-1.187-0.349]$ & Poor \\
\hline
\end{tabular}

Agreement as suggested by Cicchetti [15] (values below 0.40 are considered as "poor" agreement, between 0.40 and 0.59 as "fair" agreement, between 0.60 and 0.74 as "good" agreement and for greater 0.74 , the level of agreement is "excellent")

ICC intraclass correlation coefficient, $\mathrm{Cl}$ confidence interval, TVD total vessel density, PVD perfused vessel density, PPV proportion of perfused vessels

experience concerning the technical analysis. For these reasons, a reliable automatic analysis of the microcirculation would represent a major advantage for its clinical impact.

In the present study, macrohaemodynamic measurements after blood withdrawal revealed the typical signs of hypovolaemic shock, namely decreases in MAP, CI and SVI accompanied with an increase in heart rate. Simultaneously, lactate levels increased which might have been caused by a deranged microcirculation due to the induction of haemorrhagic shock on the one hand, but also by catecholamine release, mitochondrial and cellular dysfunction on the other hand. The decrease in haemoglobin concentration may best be explained by haemodilution due to endogenous recruitment of interstitial fluid into the intravascular compartment.

Manual analysis of the microcirculation showed results concordant with macrohaemodynamic features of hypovolaemic shock. TVD remained constant during the induction of haemorrhagic shock, and PVD and PPV were reduced. These observations support those of other investigators in clinical and preclinical studies of microcirculatory changes in haemorrhagic shock $[18,19]$. The automatic analysis, however, was not able to demonstrate these characteristic microcirculatory changes of haemorrhagic shock.

Table 4 Bland-Altman analysis between manual and automatic analyses

\begin{tabular}{lllll}
\hline Variable & Data set & Number & Mean bias [95\% Cl] & LOA \\
\hline TVD $\left[\mathrm{mm}^{*} \mathrm{~mm}^{-2}\right]$ & All videos & 85 & $7.44[6.12-8.77]$ & $-4.57-19.45$ \\
& Baseline & 41 & $7.66[5.66-9.67]$ & $-4.79-20.11$ \\
& Shock & 44 & $7.24[5.42-9.06]$ & $-4.50-18.98$ \\
PVD $\left[\mathrm{mm}^{*} \mathrm{~mm}^{-2}\right]$ & All videos & 85 & $3.37[1.61-5.12]$ & $-12.58-19.32$ \\
& Baseline & 41 & $5.61[3.45-7.78]$ & $-7.84-19.06$ \\
& Shock & 44 & $1.27[-1.38-3.93]$ & $-15.84-18.38$ \\
PPV [\%] & All videos & 85 & $-23.09[-29.66-(-16.51)]$ & $-82.83-36.65$ \\
& Baseline & 41 & $-11.62[-17.34-(-5.91)]$ & $-47.12-23.88$ \\
& Shock & 44 & $-33.77[-44.60-(-22.94)]$ & $-130.57-36.03$ \\
\hline
\end{tabular}

LOA limits of agreement, TVD total vessel density, PVD perfused vessel density, PPV proportion of perfused vessels 

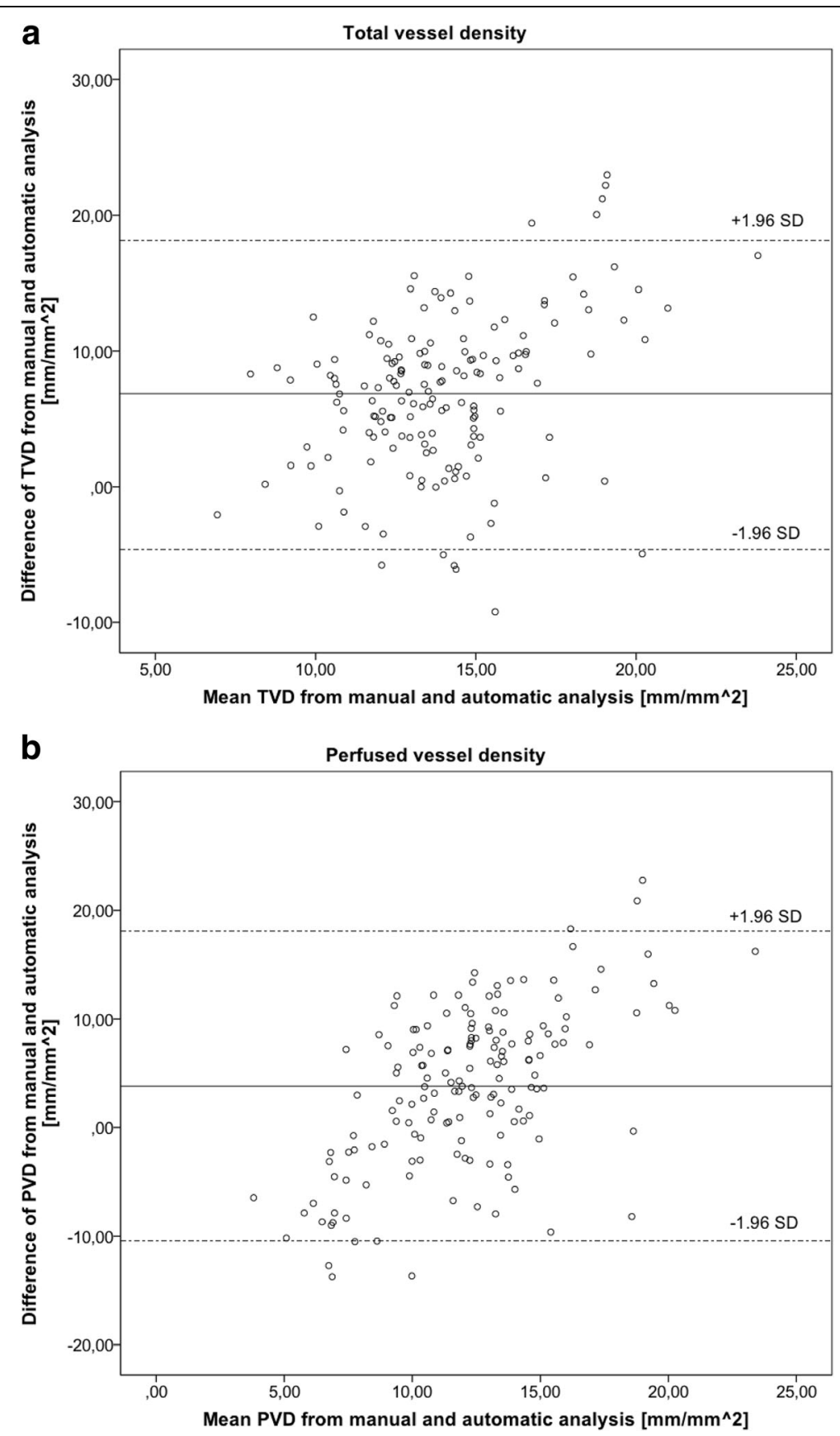

Fig. 1 Bland-Altman plots for a total vessel density and $\mathbf{b}$ perfused vessel density (each $n=85$ ). Bland and Altman recommended plotting the mean of two measurement methods against the difference of both [17]. Continuous line represents the mean difference whereas upper and lower dashed lines represent the limits of agreement (LOA) (equivalent to $\pm 1.96 \mathrm{SD}$ of mean difference). Bland-Altman plots have to be inspected visually. Mean difference represents the mean bias between measurements. LOA are a measure of dispersion between both methods. The evaluator has to decide whether these values are acceptable in the context of the measured variable. TVD total vessel density, PVD perfused vessel density, SD standard deviation, LOA limits of agreement

Examination of the Bland-Altman plots revealed a bias in the same variables whose dimension was high. Furthermore, LOA were too wide for all variables. According to Bland and Altman, these signs not only indicate a constant bias between the two methods but also a high variation making a correlation between them unlikely [17]. 
Accordingly, the comparison of the two methods evaluated by ICC revealed a poor agreement in all TVD, PVD and PPV.

Microcirculatory changes in haemorrhagic shock potentially could have influenced the correlation analysis. There is a risk that vessels, which were intermittently (e.g. large plasma gaps) or very slowly perfused by red blood cells, are better recognised by an experienced operator than by an automatic analysis software. Therefore, the correlation at baseline (when perfusion is supposed to be physiological) might be better than in haemorrhagic shock, where low flow vessels are more likely to be found. To exclude this suspicion, the agreement between manual and automatic analyses was investigated separately for each time point (baseline and shock). Notably, no correlation or agreement between the two analysis methods was found when separating the values for baseline and shock. As a consequence, an influence of the pathophysiological changes during haemorrhagic shock on the correlation seems to be unlikely.

The poor correlation between both analyses could reasonably be attributed to shortcomings of the analysis algorithm for vessel detection in the automatic analysis as illustrated in Fig. 2. Figure 2 shows screenshots of the capillary networks drawn by automatic analysis and manually by an experienced user in a corresponding video of ovine conjunctival microcirculation. The automatic analysis algorithm was not able to differentiate between different focus depths meaning that the algorithm detects vessels, which were located outside the focal plane, and, in contrast, would not be "drawn in" by an experienced investigator using manual analysis software. This misinterpretation by automatic analysis leads to different values for vessel-density-dependent variables compared to manual analysis. Further improvement of automatic analysis software will primarily have to challenge the shortcomings of vessel detection to improve overall quality of analysis results.

Parameters that describe flow conditions of the microcirculation are important as they provide information about diffusion and convection [9]. Following the consensus recommendations, evaluating the microcirculation should include a flow index [11]. An overview of flow conditions in the microcirculation is, for example, provided by the semi-quantitative microvascular flow index (MFI) by classifying microvascular flow into different categories (no, intermittent, sluggish or continuous flow) [20]. The automatic analysis provides no flow-related results except PPV and PVD, which are estimates of the flow-dependent functional capillary density, but provide no information about the flow itself. Thus, it was not possible to carry out a comparison of variables describing flow between manual analysis and automatic analysis. The missing detection of different flow states by automatic analysis would also be a likely explanation for the lack of changes in PVD and PPV following haemorrhage as detailed above. In the flowindependent parameter TVD (which simply detects all visible vessels), both analysis methods revealed no changes between baseline and shock. Manual analysis was able to detect changes in the flow-dependent variables PVD and PPV whereas automatic analysis failed to detect these.

The present study has some limitations that need to be mentioned. As videos were obtained in an animal model of haemorrhagic shock, results may not apply to human microcirculatory analysis. In addition, videos of ovine conjunctival microcirculation were investigated, whereas the sublingual region is usually the area of choice for the clinical examination of human microcirculation (especially for studying abnormalities 


\section{a}

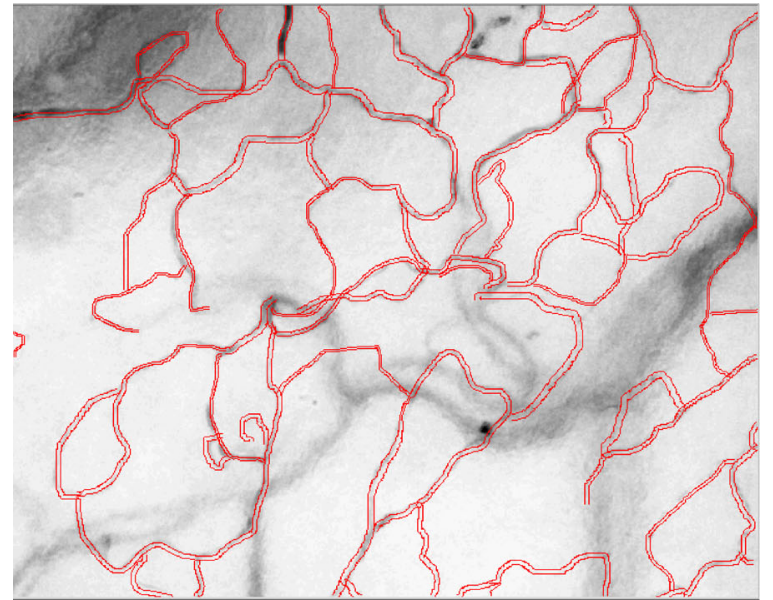

b

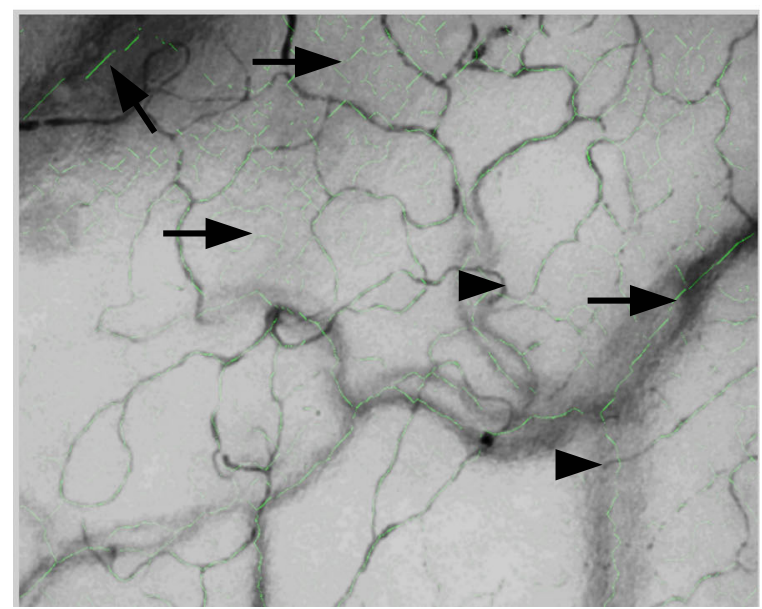

Fig. 2 Screenshot of capillary networks analysed $\mathbf{a}$ manually and $\mathbf{b}$ automatically. Screenshot of the same video: $\mathbf{a}$ analysed manually by an experienced user with the AVA software version 3.2 and $\mathbf{b}$ analysed automatically by the AVA software version 4.2. Borders of vessels in panel a are coloured red, and centre lines of vessels in panel $\mathbf{b}$ are coloured lime green. Marks in panel $\mathbf{b}$ show examples of differences from automatic to manual analysis: Arrowheads $=$ no vessel detected compared to manual analysis; arrows $=$ more vessels detected as by manual analysis

in sepsis or haemorrhagic shock) $[2,11,19]$. The reason for choosing the sublingual region is, on the one hand, its easy accessibility and, on the other hand, its blood perfusion is based on proximity to the central arteries. As the same arguments regarding vascular anatomy and accessibility apply to the conjunctival microcirculation, it may also be a valuable target. The good correlation between sublingual and conjunctival microcirculation has recently been demonstrated in an experimental study by Yin et al. [21]. Based on our own experience, video quality of ovine conjunctival microcirculation videos is superior to the quality of videos derived from ovine sublingual microcirculation due to anatomical conditions such as a long floppy tongue vs. large eyeballs with easy access. Thus, we decided to perform a comparison between manual and automatic 
analyses using high-quality conjunctival videos. It may be possible that automatic analysis works better with sublingual videos of microcirculation. Also, the AVA software version 4.2 was developed to automatically analyse videos obtained by SDF imaging technique. However, as microcirculatory videos obtained by IDF technique provide better image quality $[22,23]$ and can be converted to and opened in AVA software version 4.2 , analysis results should be at least as good as with videos from SDF cameras. At last, it is unclear if the results of the present study can be transferred to pathophysiological conditions other than haemorrhagic shock as we only examined microcirculation during the induction of haemorrhage. Further investigations are needed to address these limitations.

\section{Conclusions}

Characteristic changes in microcirculation during ovine haemorrhagic shock could not be reproduced by automatic analysis with the AVA 4.2 software. No flow-quantifying variable was given by automatic analysis, and there was only a poor correlation between automatic analysis and the current gold standard (manual analysis). Therefore, the automatic analysis with the AVA 4.2 software cannot be recommended at present. Further improvements in the detection algorithm are necessary, before an automatic analysis could be reevaluated for the evaluation of microcirculation in research or clinical routine.

\section{Additional files}

Additional file 1: Raw data of blood withdrawal, haemodynamics, blood gas analysis and microcirculatory analysis. (XLSX $10 \mathrm{~kb}$ )

Additional file 2: Raw data of microcirculatory analysis of individual videos. (XLSX $14 \mathrm{~kb}$ )

\section{Abbreviations}

Cl: cardiac index; HR: heart rate; ICC: intraclass correlation coefficient; IDF: incident dark field; LOA: limits of agreement; MAP: mean arterial pressure; PPV: proportion of perfused vessels; PVD: perfused vessel density; SDF: sidestream dark field; SVI: stroke volume index; TVD: total vessel density

\section{Acknowledgements}

Not applicable.

\section{Funding}

The study providing the data for the present investigation was an investigator-initiated trial supported by Fresenius Kabi, Bad Homburg, Germany.

\section{Availability of data and materials}

The datasets supporting the conclusions of this article are included within the article and its additional files.

\section{Authors' contributions}

PA and $\mathrm{MH}$ helped in the design and conception, data acquisition, data management, interpretation of data, statistical analysis and preparation of the manuscript. TK, AM and SR helped in the data management and interpretation of data, revised the manuscript critically and approved the final version. HVA performed the data acquisition and interpretation of data, revised the manuscript critically and approved the final version. MW helped in the design and conception and made profound revision of the manuscript and approved the final version. CE also helped in the design and conception, data acquisition, data management, interpretation of data and preparation of the manuscript. All authors read and approved the final manuscript.

\section{Competing interests}

The authors declare that they have no competing interests.

\section{Consent for publication}

Not applicable.

Ethics approval

The present study was approved by the local veterinary authority (North Rhine-Westphalia State Environment Agency) under reference number 84-02.04.2013.A003. 


\section{Author details}

'Department of Anaesthesiology, Intensive Care and Pain Therapy, Muenster University Hospital, Albert-Schweitzer-Campus 1, Building A1, 49149 Muenster, Germany. ${ }^{2}$ Department of Cardiovascular, Respiratory, Nephrological, Anesthesiological and Geriatric Sciences, University of Rome, "La Sapienza", Viale del Policlinico 155, 00161 Rome, Italy. ${ }^{3}$ Department of Anaesthesiology, Greifswald University Hospital, Ferdinand-Sauerbruch-Straße, 17475 Greifswald, Germany.

\section{Received: 9 August 2016 Accepted: 7 November 2016}

\section{Published online: 18 November 2016}

\section{References}

1. Edul VSK, Ince C, Vazquez AR et al (2016) Similar microcirculatory alterations in patients with normodynamic and hyperdynamic septic shock. Ann Am Thorac Soc 13:240-247. doi:10.1513/AnnalsATS.201509-6060C

2. Edul VSK, Enrico C, Laviolle B et al (2012) Quantitative assessment of the microcirculation in healthy volunteers and in patients with septic shock. Crit Care Med 40:1443-1448. doi:10.1097/CCM.0b013e31823dae59

3. Trzeciak S, Dellinger RP, Parrillo JE et al (2007) Early microcirculatory perfusion derangements in patients with severe sepsis and septic shock: relationship to hemodynamics, oxygen transport, and survival. Ann Emerg Med 49:88-98. doi:10.1016/j.annemergmed.2006.08.021, 98.e1-2

4. Sakr Y, Dubois M-J, De Backer D et al (2004) Persistent microcirculatory alterations are associated with organ failure and death in patients with septic shock. Crit Care Med 32:1825-1831

5. Vellinga NAR, Boerma EC, Koopmans M et al (2015) International study on microcirculatory shock occurrence in acutely ill patients. Crit Care Med 43:48-56. doi:10.1097/CCM.0000000000000553

6. Goedhart PT, Khalilzada M, Bezemer R et al (2007) Sidestream dark field (SDF) imaging: a novel stroboscopic LED ring-based imaging modality for clinical assessment of the microcirculation. Opt Express 15:15101-15114

7. Aykut G, Veenstra G, Scorcella C et al (2015) Cytocam-IDF (incident dark field illumination) imaging for bedside monitoring of the microcirculation. Intensive Care Med Exp 3:40. doi:10.1186/s40635-015-0040-7

8. Tanaka S, Harrois A, Nicolaï C et al (2015) Qualitative real-time analysis by nurses of sublingual microcirculation in intensive care unit: the MICRONURSE study. Crit Care 19:388. doi:10.1186/s13054-015-1106-3

9. Ince C (2015) Hemodynamic coherence and the rationale for monitoring the microcirculation. Crit Care 19(Suppl 3):S8. doi:10.1186/cc14726

10. Kanoore Edul VS, Ince C, Dubin A (2015) What is microcirculatory shock? Curr Opin Crit Care 21:245-252. doi:10.1097/MCC.0000000000000196

11. De Backer D, Hollenberg S, Boerma C, et al. (2007) How to evaluate the microcirculation: report of a round table conference. In: Crit Care. p R101

12. Hansard SL (1956) Residual organ blood volume of cattle, sheep and swine. Proc Soc Exp Biol Med 91:31-34

13. Massey MJ, Larochelle E, Najarro G et al (2013) The microcirculation image quality score: development and preliminary evaluation of a proposed approach to grading quality of image acquisition for bedside videomicroscopy. J Crit Care 28:913-917. doi:10.1016/j.jcrc.2013.06.015

14. Dobbe JGG, Streekstra GJ, Atasever B et al (2008) Measurement of functional microcirculatory geometry and velocity distributions using automated image analysis. Med Biol Eng Comput 46:659-670. doi:10.1007/s11517-008-0349-4

15. Rankin G, Stokes M (1998) Reliability of assessment tools in rehabilitation: an illustration of appropriate statistical analyses. Clin Rehabil 12:187-199

16. Cicchetti DV (1994) Guidelines, criteria, and rules of thumb for evaluating normed and standardized assessment instruments in psychology. Psychol Assess 6:284-290. doi:10.1037/1040-3590.6.4.284

17. Bland JM, Altman DG (1986) Statistical methods for assessing agreement between two methods of clinical measurement. Lancet 1:307-310

18. González R, Urbano J, López J et al (2016) Microcirculatory alterations during haemorrhagic shock and after resuscitation in a paediatric animal model. Injury 47:335-341. doi:10.1016/j.injury.2015.10.075

19. Tachon G, Harrois A, Tanaka S et al (2014) Microcirculatory alterations in traumatic hemorrhagic shock. Crit Care Med 42:1433-1441. doi:10.1097/CCM.0000000000000223

20. Boerma EC, Mathura KR, van der Voort PHJ et al (2005) Quantifying bedside-derived imaging of microcirculatory abnormalities in septic patients: a prospective validation study. Crit Care 9:R601-R606. doi:10.1186/cc3809

21. Yin L, Yang Z, Yu H et al (2016) Changes in sublingual microcirculation is closely related with that of bulbar conjunctival microcirculation in a Rat model of cardiac arrest. Shock 45:428-433. doi:10.1097/SHK.0000000000000508

22. van Elteren HA, Ince C, Tibboel D et al (2015) Cutaneous microcirculation in preterm neonates: comparison between sidestream dark field (SDF) and incident dark field (IDF) imaging. J Clin Monit Comput 29:543-548. doi:10.1007/s10877-015-9708-5

23. Gilbert-Kawai E, Coppel J, Bountziouka $V$ et al (2016) A comparison of the quality of image acquisition between the incident dark field and sidestream dark field video-microscopes. BMC Med Imaging 16:10. doi:10.1186/s12880-015-0078-8 